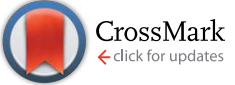

Cite this: RSC Adv., 2017, 7, 9880
Received 2nd December 2016 Accepted 22nd January 2017

DOI: 10.1039/c6ra27666k

rsc.li/rsc-advances

\section{An ultrafiltration membrane with enhanced photocatalytic performance from grafted $\mathrm{N}-\mathrm{TiO}_{2} /$ graphene oxide}

\author{
Wei Chen, ${ }^{\text {ab }}$ Ting Ye, ${ }^{b}$ Hang Xu, ${ }^{\star a b}$ Taoyuan Chen, ${ }^{b}$ Nannan Geng ${ }^{b}$ \\ and Xiaohong Gao ${ }^{\mathrm{b}}$
}

An enhanced photocatalytic ultrafiltration membrane was prepared by grafting with $\mathrm{N}-\mathrm{TiO}_{2} /$ graphene oxide. After $\mathrm{N}-\mathrm{TiO}_{2} /$ graphene oxide particles and a polysulfone membrane had been prepared, the $\mathrm{N}-\mathrm{TiO}_{2} /$ graphene oxide was distributed in deionized water, poured onto the membrane surface and grafted onto the membrane surface by using a pump filter. Scanning electron microscopy and atomic force microscopy were used to investigate the surface and morphological structure of the prepared membranes. Fourier transform infrared spectroscopy and X-ray photoelectron spectroscopy were used to analyze the elemental compositions and chemical bonds of the membranes. The hydrophilicity of the membrane surface was investigated by a sessile-drop method. The membrane water flux was tested in an ultrafiltration cup system. Methylene blue removal under darkness, ultraviolet light and sunlight were used to characterize the photocatalytic ability. The photocatalytic membrane exhibited an enhanced photocatalytic performance, especially in sunlight rather than in ultraviolet light. The synergistic effect of photocatalysis and filtration was tested; the photocatalytic membrane exhibited a better methylene-blue removal ability than a pure membrane as the methylene blue concentration in filtered water was lower. The recyclability of the photocatalytic membrane had a great improvement compared with the powder photocatalyst.

\section{Introduction}

In photocatalytic oxidation technology, which occurs under the effect of light, a substance absorbs light energy and is stimulated to cause molecular excitation. This results in hydroxyl freeradical generation with strong oxidizing properties on the photocatalyst surface and organic pollutant degradation. ${ }^{\mathbf{1}}$ Titanium dioxide $\left(\mathrm{TiO}_{2}\right)$ is a semiconductor and is termed a green environmental protection material in the twenty-first century because of its low cost, non-toxicity, excellent chemical stability, corrosion resistance and high photocatalytic activity. ${ }^{2-4}$ However, certain disadvantages limit its practical application. The energy gap of $\mathrm{TiO}_{2}$ between the valence and conduction bands is $\sim 3.2 \mathrm{eV}$, which means that it can only be triggered by ultraviolet (UV) light $(\lambda \leq 387.5 \mathrm{~nm}),{ }^{5,6}$ which implies a utilization of only $\sim 4 \%$ of sunlight. Therefore, titanium-dioxide stimulation requires large amounts of energy that cannot be provided by the sun. Photocatalysis is most dependent on photogenerated hole oxidation and photogenerated electron reduction, but the photogenerated electron hole recombines easily, ${ }^{7,8}$ so the quantum efficiency of $\mathrm{TiO}_{2}$ is unsatisfactory. The $\mathrm{TiO}_{2}$ photocatalytic

${ }^{a}$ Key Laboratory of Integrated Regulation and Resource Development on Shallow Lakes of Ministry of Education, College of Environment, Hohai University, Nanjing 210098, China. E-mail: xuhang810826@163.com; Tel: +8613770848137

${ }^{b}$ College of Environment, Hohai University, Nanjing 210098, China ability and its particle size are inversely proportional. Although the preparation of small $\mathrm{TiO}_{2}$ is advantageous, such a small particle size is difficult to recycle, ${ }^{4,9} \mathrm{TiO}_{2}$ use in practice would be limited if there were no way to recycle the $\mathrm{TiO}_{2}$. To solve these problems, $\mathrm{TiO}_{2}$ modification is being researched worldwide.

Current commonly used $\mathrm{TiO}_{2}$ modification methods include heavy-metal modification, ${ }^{\mathbf{1 0}}$ metal modification, ${ }^{\mathbf{1 1}}$ non-metal modification, ${ }^{12}$ dye-sensitized modification, ${ }^{13}$ semiconductorcompound modification ${ }^{\mathbf{1 4}}$ and polyoxometalate modification. ${ }^{\mathbf{1 5}}$ Nitrogen-doping of $\mathrm{TiO}_{2}$ is a non-metal modification that replaces oxygen vacancies with nitrogen ${ }^{12,16}$ or introduces nitrogen to $\mathrm{TiO}_{2}$ interstitially, ${ }^{3}$ is efficient in reducing the energy gap between valence and conduction bands and is considered a promising photocatalyst to increase photocatalytic efficiency. Graphene comprises a single sheet of $\mathrm{sp}^{2}$-hybridized carbon atoms in a hexagonal lattice and has received increased attention in various fields because of its high surface area and attractive electronic, thermal, mechanical and optical properties. ${ }^{\mathbf{1 1 1 , 1 6}}$ Graphene oxide (GO) is a group of oxygen-containing functional groups (hydroxyl groups, carboxyl groups, epoxy groups and etc.) that is based on a graphene structure. ${ }^{17}$ The effect of GO on photocatalytic activity enhancement has been researched, in which GO acts as an electron acceptor ${ }^{\mathbf{1 1 8}}$ to hinder photogenerated electron-hole pair recombination. GO behaves as an impurity ${ }^{\mathbf{1 1 , 1 9}}$ to form $\mathrm{Ti}-\mathrm{O}-\mathrm{C}^{\mathbf{2 0}}$ bonds, and therefore expands light absorption to sunlight. ${ }^{21}$ 
To solve difficulties in photocatalyst recycle, the embodiment of photocatalysts in some support materials (such as glass, ceramic, activated carbon, magnetic materials and membranes $)^{\mathbf{4 , 9 , 2 2 - 2 5}}$ has been researched. Photocatalytic membranes are considered a good support option. Photocatalyst immobilization on membranes prevents loss and improves its utilization. ${ }^{26}$ Membranes modified by photocatalysts may affect various membrane properties such as water permeability, ${ }^{27}$ hydrophilicity, ${ }^{28}$ contaminant rejection ${ }^{29}$ and fouling resistance. ${ }^{30} \mathrm{GO}$ addition to photocatalytic membranes is advantageous; the hydroxyl, carbonyl and carboxylic groups of the GO make the membrane more hydrophilic and its mechanical properties may increase the membrane strength. ${ }^{31}$

Photocatalysts can be immobilized in the membrane by nesting them inside the membrane or grafting them on the surface. ${ }^{25,32}$ Nesting is achieved by photocatalyst addition into the casting membrane solution. During membrane formation, photocatalyst is distributed in the membrane. This type of photocatalytic membrane improves membrane properties (water permeability, hydrophilicity, fouling resistance). However, it is difficult to stimulate internal photocatalysts, and such photocatalytic membranes exhibit a weak photocatalytic performance. ${ }^{33}$ Photocatalysts are grafted on the membrane surface by physical or chemical binding. Such photocatalytic membranes exhibit excellent photocatalytic performance. It is difficult to deposit organics on the membrane surface because of the photocatalytic performance, and this results in a lower possibility of membrane fouling. ${ }^{25}$

Two types of study exist on this topic. $\mathrm{N}-\mathrm{TiO}_{2} /$ graphene oxide and modified $\mathrm{TiO}_{2}$ has been prepared as a powder and exhibits a superior photocatalytic performance, ${ }^{5,7}$ however, hardly can these photocatalysts be recycled. In other studies, the membrane surface has been modified with $\mathrm{TiO}_{2} /$ graphene oxide, ${ }^{\mathbf{2 4 , 2 5 , 3 4}}$ but its photocatalytic performance was better in UV light. To our best knowledge, the use of $\mathrm{N}-\mathrm{TiO}_{2} /$ graphene oxide to produce photocatalytic membranes has not been reported previously. In this study, a polysulfone-based ultrafiltration membrane was prepared by grafting $\mathrm{N}-\mathrm{TiO}_{2} /$ graphene oxide onto the membrane (NTG-M) and the membrane was characterized.

\section{Materials and methods}

\subsection{Nanoparticles and chemicals}

Tetrabutyl titanate (analytical reagent, AR, purity $\geq 98.5 \%$ ), anhydrous ethanol (AR, purity $\geq 99.7 \%$ ), urea (AR, purity $\geq$ 99.0\%), glacial acetic acid (AR, purity $\geq 99.5 \%$ ), hydrochloric acid ( $\mathrm{HCl}, 6 \mathrm{M})$, polysulfone (AR, purity $\left.\geq 99 \%, M_{\mathrm{r}}=1327.58\right)$, $N$-methylpyrrolidone (NMP, AR, purity $\geq 99 \%$ ), polyethylene glycol (PEG 600, AR), polyvinylpyrrolidone (PVP, guarantee reagent, GR) and methylene blue ( $\mathrm{MB}$, purity $\geq 98.5 \%$ ) were from Sinopharm Chemical Reagent Co. Ltd. (Shanghai, China). Graphene oxide (GO, powder, diameter $=0.5-5 \mu \mathrm{m}$, thickness $=$ 0.6-1 nm, purity $\geq 99.5 \%$ ) was from Hengqiu Graphene Technology Co. Ltd. (SuZhou, China). Deionized water (DI) was made using a Milli-Q integral system.

\section{$2.2 \mathrm{~N}-\mathrm{TiO}_{2}(\mathrm{NT})$ preparation}

NT nanoparticles were prepared by using the sol-gel approach. Tetrabutyl titanate $(10.0 \mathrm{~mL})$ was added slowly into anhydrous ethanol $(40.0 \mathrm{~mL})$ to form solution A. Urea was dissolved in a solution of anhydrous ethanol $(10.0 \mathrm{~mL})$, DI $(4.0 \mathrm{~mL})$ and glacial acetic acid $(2.0 \mathrm{~mL})$ at $\mathrm{pH} 2.0$ (adjusted using $\mathrm{HCl}$ ) with stirring to form solution B. Solution B was added dropwise into solution A with vigorous stirring. The solution was stirred for a further $0.5 \mathrm{~h}$ before allowing it to settle for $\sim 3 \mathrm{~h}$ to form a gel. The solution was dried at $100{ }^{\circ} \mathrm{C}$ for $\sim 24 \mathrm{~h}$ to form yellow crystals. The solids were ground to a white powder and calcined at $500{ }^{\circ} \mathrm{C}$ for $2 \mathrm{~h}$, and NT was formed. The $\mathrm{TiO}_{2}$ preparation was the same as for the NT but without urea addition.

\section{$2.3 \mathrm{~N}-\mathrm{TiO}_{2} / \mathrm{GO}(\mathrm{NTG})$ preparation}

NTG was prepared by a hydrothermal method. GO (0.025 g) was dispersed in $80 \mathrm{~mL}$ of DI with ultrasonic treatment for $2 \mathrm{~h}$, and then $\mathrm{TiO}_{2}(0.475 \mathrm{~g})$ was added into the suspension for an additional $1 \mathrm{~h}$ of ultrasonic treatment. Soon afterwards, the suspension was moved to a $100 \mathrm{~mL}$ Teflon-lined stainless autoclave with the $\mathrm{pH}$ being adjusted to 3-4 (using $\mathrm{HCl}$ ). After the reactor had been kept at $180{ }^{\circ} \mathrm{C}$ for $15 \mathrm{~h}$, the mixture was cooled to room temperature naturally. NTG was obtained by freeze drying. $\mathrm{TiO}_{2} / \mathrm{GO}$ (TG) was prepared by the same process except for the difference in starting materials where $\mathrm{TiO}_{2}$ was used to prepare the TG and NT was used for the NTG.

\subsection{Polysulfone membrane preparation}

Polysulfone (18.0 g) was dried and added into NMP (80.0 g) solution with continuous stirring for $5 \mathrm{~h}$ at $50{ }^{\circ} \mathrm{C}$. PEG $(1.6 \mathrm{~g})$ and PVP ( $0.4 \mathrm{~g})$ were added to the solution with further stirring for $0.5 \mathrm{~h}$. The solution was maintained in a drying atmosphere for $\sim 24 \mathrm{~h}$ to release bubbles. The solution was poured on a spotless glass plate, and using a $100 \mu \mathrm{m}$ casting knife and with a constant casting rate, a cast film was formed. The cast film was immediately transferred into a non-solvent coagulation bath (DI at $25{ }^{\circ} \mathrm{C}$ ). The membrane were washed with DI to remove residual solvent and preserved in DI for at least $24 \mathrm{~h}$ before it was used.

\subsection{NTG-M preparation}

NTG $(0.05 \mathrm{~g})$ was dispersed in $100 \mathrm{~mL}$ DI by ultrasonic treatment to form $0.5 \mathrm{~g} \mathrm{~L}^{-1}$ NTG solution. NTG solution $(20 \mathrm{~mL})$ was poured onto the membrane surface using a pump filter. $\mathrm{N}-\mathrm{TiO}_{2} /$ graphene oxide particles were grafted on the membrane surface while the DI was filtered. The NTG-M was dried at $50{ }^{\circ} \mathrm{C}$, washed with DI and preserved in DI at least $24 \mathrm{~h}$ before use. Membranes grafted using $\mathrm{TiO}_{2}$ (T-M), NT (NT-M) and TG (TG-M) were prepared by the same process except for differences in starting materials.

\subsection{Characterization}

The surface and morphology of the prepared membranes were studied by scanning electron microscopy (SEM, Zeiss Ultra 55, Oberkochen, Germany) and atomic force microscopy (AFM, 

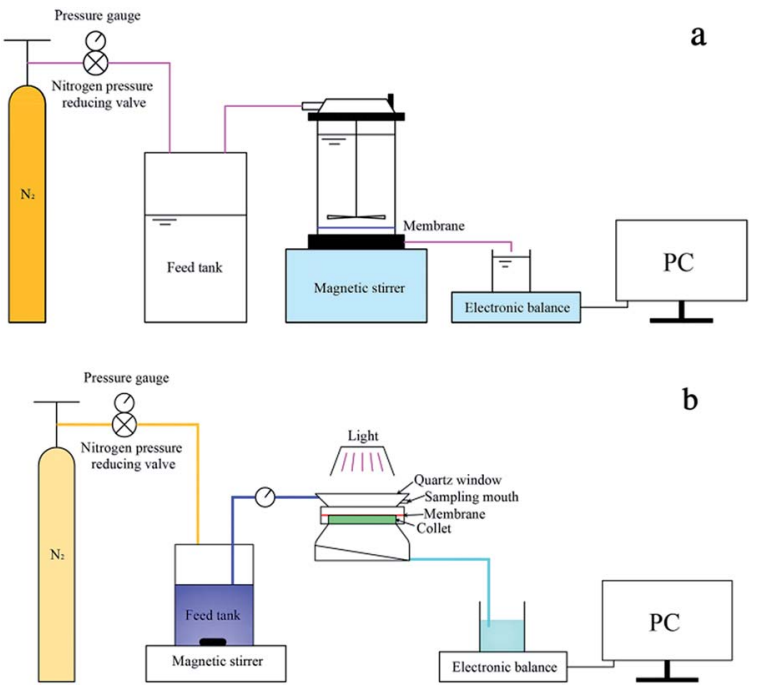

Fig. 1 Schematic diagram of (a) ultrafiltration cup system and (b) equipment to measure the removal of $M B$

Multimode 8, Bruker, Santa Barbara, CA, USA). The elemental compositions and chemical bonds of the membranes were analyzed by using Fourier transform infrared spectroscopy (FTIR, Nicolet 6700, Thermo Scientific, Waltham, MA, USA) and Xray photoelectron spectroscopy (XPS, K-Alpha, Thermo Scientific, West Palm Beach, FL, USA). The membrane surface hydrophilicity was studied by the sessile-drop method (G10, Kruss, Hamburg, Germany).

The membrane water flux was tested in an ultrafiltration cup system as illustrated in Fig. 1(a). The system consisted of a nitrogen gas cylinder to provide pressure, a feed tank to avoid backflow of liquid, an ultrafiltration cup as the core part of the system and an electronic balance to measure filtered water. The synergistic effect of photocatalysis and filtration of the NTG-M was measured by the removal of $\mathrm{MB}$ in the equipment shown in Fig. 1(b), with attachments that were similar to the ultrafiltration cup system but differed at the core. The core contained a light source, a sealed quartz glass container with a water inlet and a sampling mouth, and a collet to support the membrane.

\section{Results and discussion}

\subsection{Membrane characterization}

SEM (Fig. 2) was used to assess the membrane surface modification of NTG-M and other prepared membranes (M, T-M, NTM, TG-M). Fig. 2(a) shows a pure membrane with nothing grafted on its surface. The top surface of T-M and NT-M are shown in Fig. 2(b) and (c), $\mathrm{TiO}_{2}$ and NT are distributed evenly on the membrane surface but some $\mathrm{TiO}_{2}$ (NT) recombines. The morphologies of TG-M and NTG-M are shown in Fig. 2(d) and (e), the arrangement of the TG (NTG) platelets leads a good $\mathrm{TiO}_{2}$ (NT) distribution on the GO sheets, which increases the $\mathrm{TiO}_{2}$ (NT) surface area and the photocatalytic performance of the corresponding photocatalytic membranes. Fig. 2(f) shows the SEM image of NTG, the mean particle size of NT is about 30$50 \mathrm{~nm}$. The weigh difference between membranes before and

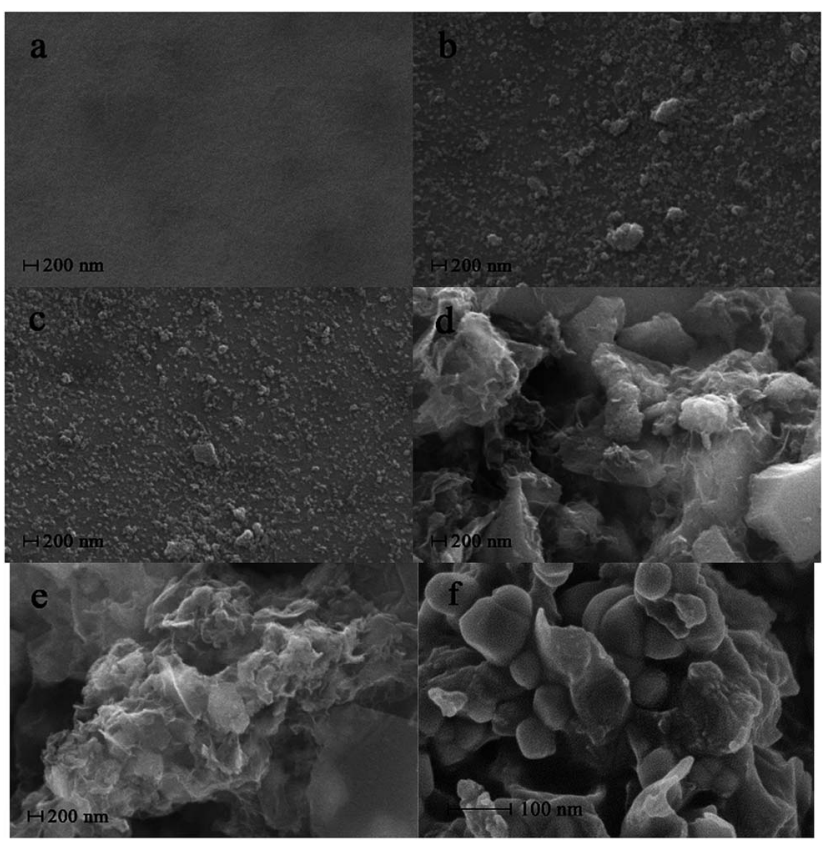

Fig. 2 Top surface SEM images of (a) M, (b) T-M, (c) NT-M, (d) TG-M, (e) NTG-M and (f) SEM image of NTG.

after grafting NTG is $\sim 9 \mathrm{mg}$, which means the loading amount of NTG in NTG-M is $\sim 3 \mathrm{~g} \mathrm{~m}^{-2}$.

Fig. 3 shows the three-dimensional surface AFM images of M, T-M, NT-M, TG-M and NTG-M at a scan size of $5 \mu \mathrm{m} \times 5$ $\mu \mathrm{m}$. The brightest regions represent the highest peaks of the membrane surface, the dark areas on the membrane are the pores or lowest valley. The surface-roughness parameters (mean roughness $S_{\mathrm{a}}$, root mean square of $Z$ data $S_{\mathrm{q}}$, height difference between the highest peak and the lowest valley $S_{\mathrm{y}}$ ) of bare and grafted membranes are listed in Table 1 . These surfaceroughness parameters are connected with the ability to absorb and desorb pollutant on the membrane surface. Because photocatalysis takes place in the surface of photocatalyst, photocatalyst with higher surface area is expected to have a higher photocatalytic efficiency. NTG-M (TG-M) has a higher surface roughness than $\mathrm{M}, \mathrm{T}-\mathrm{M}$ and NT-M, we predict that NTG-M (TG-M) can absorb more pollutant and then speed up photocatalytic rate.

Fig. 4 shows the surface FT-IR spectra for M, T-M, NT-M, TG$M$ and NTG-M. Peaks at $1732 \mathrm{~cm}^{-1}$ and $3000-3600 \mathrm{~cm}^{-1}$ can be assigned to the carbonyl groups $(\mathrm{C}=\mathrm{O})$ and hydroxyl groups $(\mathrm{OH}) 16$ that appear in the spectrum of TG-M and NTG-M. Peaks at $1401 \mathrm{~cm}^{-1}, 1226 \mathrm{~cm}^{-1}$ and $1050 \mathrm{~cm}^{-1}$ can be attributed to the skeletal vibration of unoxidized graphitic, epoxy groups $\mathrm{C}-\mathrm{O}$ and the hydroxyl group stretching mode $\mathrm{C}-\mathrm{O},{ }^{35,36}$ which are stronger in the spectra of TG-M and NTG-M over those of M, T$\mathbf{M}$ and NT-M. The increase in oxygen-containing functional groups shows that membrane hydrophilicity is enhanced. These oxygen-containing functional groups (hydroxyl groups, carboxyl groups and epoxy groups) can only be derived from GO. A broad band at wavenumbers between 1000 and $450 \mathrm{~cm}^{-1}$ is linked with Ti-O-Ti and Ti-O-C. ${ }^{37}$ 

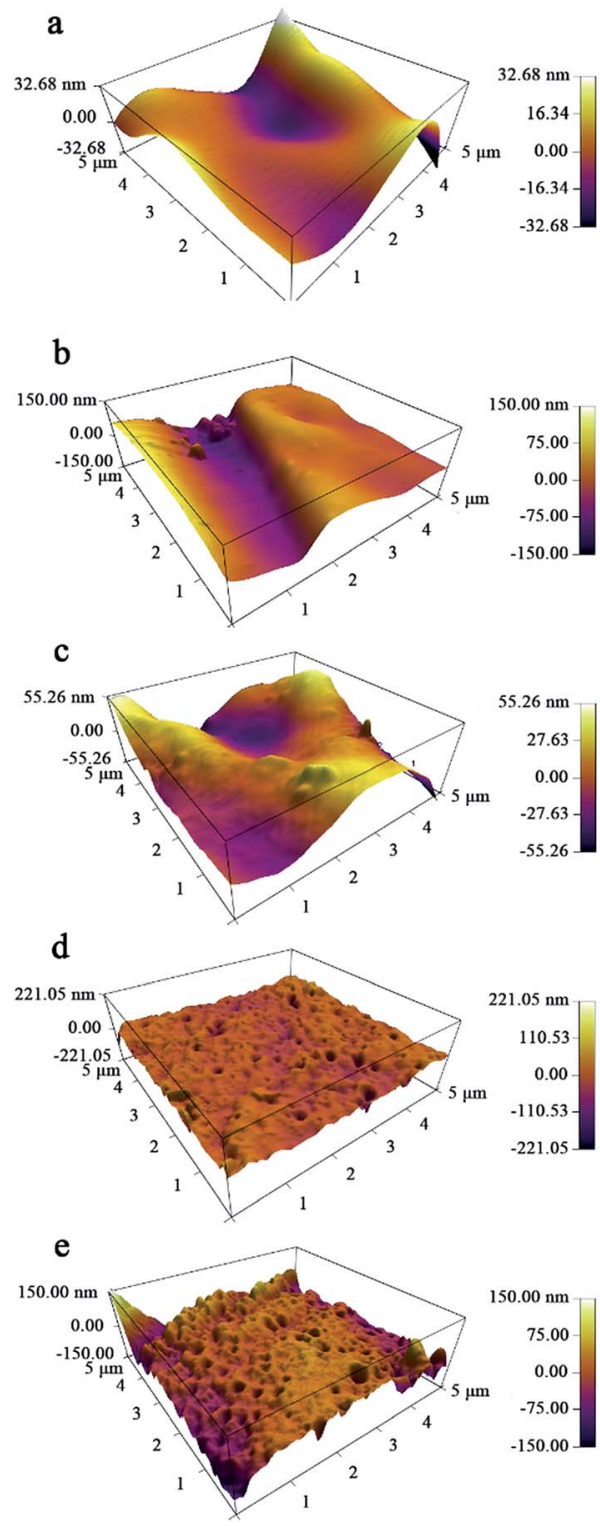

Fig. 3 Three-dimensional AFM images with a scan area of $5 \mu \mathrm{m} \times 5$ $\mu \mathrm{m}$ for (a) M, (b) T-M, (c) NT-M, (d) TG-M and (e) NTG-M.

Table 1 Surface roughness parameters of membranes that result from analyzing three randomly chosen AFM images $(5 \mu \mathrm{m} \times 5 \mu \mathrm{m})$

\begin{tabular}{lrll}
\hline & \multicolumn{3}{l}{ Roughness parameters } \\
\cline { 2 - 4 } Membrane & \multicolumn{1}{c}{$S_{\mathrm{a}}(\mathrm{nm})$} & $S_{\mathrm{q}}(\mathrm{nm})$ & \multicolumn{1}{l}{$S_{\mathrm{y}}(\mathrm{nm})$} \\
\hline M & 9.450 & 11.942 & $42.200+58.529=100.729$ \\
T-M & 13.137 & 17.167 & $73.397+62.532=135.929$ \\
NT-M & 16.505 & 20.081 & $53.325+70.392=123.717$ \\
TG-M & 16.959 & 21.332 & $111.675+164.387=276.062$ \\
NTG-M & 21.361 & 27.270 & $165.907+132.087=297.994$
\end{tabular}

$M$ and NTG-M were investigated by XPS spectra to confirm the nitrogen doping. Fig. 5 shows that peaks at 161, 284, 399, 458 and $530 \mathrm{eV}$ can be assigned to S 2p, C 1s, N 1s, Ti 2p and $\mathrm{O} 1 \mathrm{~s}$, respectively. The atomic percentages of $\mathrm{S} 2 \mathrm{p}, \mathrm{C} 1 \mathrm{~s}, \mathrm{~N} 1 \mathrm{~s}$,

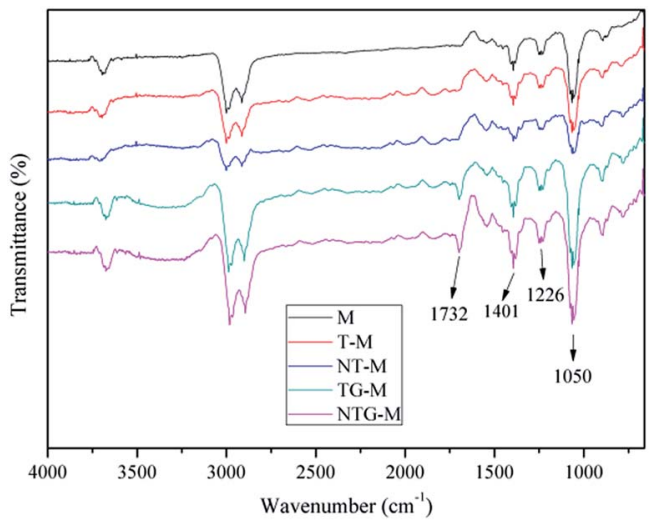

Fig. 4 FT-IR spectra of $M, T-M, N T-M, T G-M$ and NTG-M.

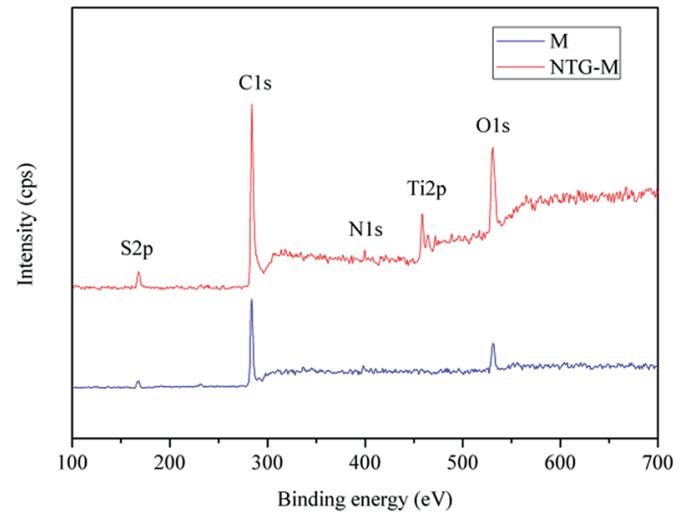

Fig. 5 XPS spectra of M and NTG-M.

Ti $2 \mathrm{p}$ and $\mathrm{O}$ 1s in NTG-M are 6.62 at\%, 74.54 at\%, 0.55 at\%, 2.47 at $\%$ and 15.82 at\%, respectively, whereas their atomic percentages in $\mathrm{M}$ are 7.51 at\%, 77.49 at\%, 0.22 at\%, 0.00 at\% and 14.78 at\%. The small amount of $\mathrm{N} 1 \mathrm{~s}$ that is contained in $\mathrm{M}$ may originate from membrane fabrication because polysulfone was dissolved in NMP that contains $\mathrm{N}$. The percentage of $\mathrm{N}$ in NTG-M is higher than in pure membranes, and can originate only from NTG, which indicates that nitrogen was doped on $\mathrm{TiO}_{2}$. With the grafting of NTG on the membrane, the percentages of $\mathrm{C}$ and $\mathrm{S}$ were reduced, whereas $\mathrm{Ti}$ and $\mathrm{O}$ increased because the main ingredient in NTG is $\mathrm{TiO}_{2}$.

Contact angle measurements are shown in Fig. 6 (M and NTG-M) and Table 2 (M, T-M, NT-M, TG-M and NTG-M). In Fig. 6(a) and (b), once a bare membrane had been grafted by NTG, the apparent pure water-contact angle decreased from $81^{\circ}$ to $64^{\circ}$, which represented a much higher affinity between the membrane surface and water because of the hydrophilicity of $\mathrm{TiO}_{2}$ and oxygen-containing functional groups in GO. The contact angle of NTG-M improved slightly compared with TG-M, and was $\sim 65^{\circ}$, which implies that nitrogen doping increases the $\mathrm{TiO}_{2}$ hydrophilicity and then increases the hydrophilicity of the grafted membranes. 


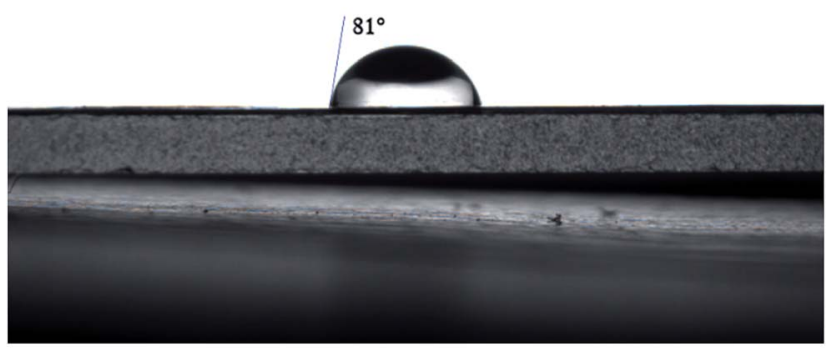

b

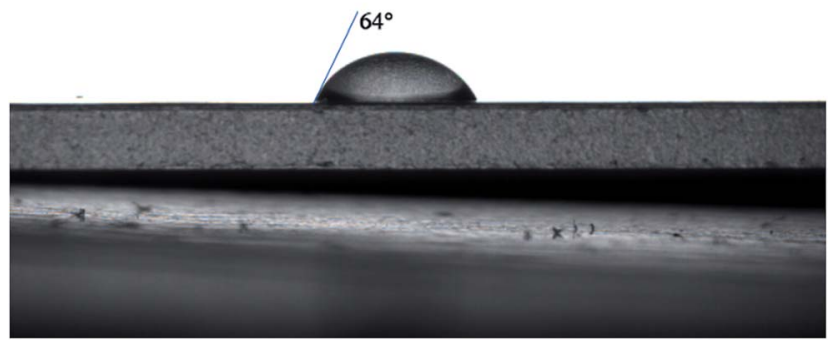

Fig. 6 Water contact angle of (a) M and (b) NTG-M.

Table 2 Contact angle and water flux of M, T-M, NT-M, TG-M and NTG-M

\begin{tabular}{llc}
\hline Membrane & Contact angle $\left(^{\circ}\right)$ & Water flux $\left(\mathrm{L} \mathrm{m}^{-2} \mathrm{~h}^{-1}\right)$ \\
\hline M & $81 \pm 1.5$ & $108 \pm 4.2$ \\
T-M & $78 \pm 1.4$ & $78 \pm 3.3$ \\
NT-M & $76 \pm 1.9$ & $82 \pm 3.0$ \\
TG-M & $65 \pm 2.2$ & $66 \pm 3.9$ \\
NTG-M & $64 \pm 1.8$ & $70 \pm 2.5$
\end{tabular}

The pure water flux of membranes was tested in the system as illustrated in Fig. 1(a) at 0.1 MPa and at room temperature, and the results are shown in Table 2 . The pure water flux for a pure membrane is $108 \mathrm{~L} \mathrm{~m}^{-2} \mathrm{~h}^{-1}$, and this value decreases after grafting by $\mathrm{TiO}_{2}, \mathrm{NT}$, TG and NTG, respectively. T-M exhibits a decrease in pure water flux by approximately $28 \%$ relative to the water flux of $\mathrm{M}$ because of the obstruction of $\mathrm{TiO}_{2}$, which recombined partly on the surface. TG-M has an additional 15\% membrane resistance to water flux compared with T$\mathrm{M}$, possibly because of the additional film thickness from the GO nanosheets. NTG-M promotes the pure water flux slightly compared with TG-M, possibly because nitrogen doping increases the $\mathrm{TiO}_{2}$ hydrophilicity and thus the pure water flux.

\subsection{Photocatalytic performance}

Without filtration performance, the photocatalytic performance of M, T-M, NT-M, TG-M and NTG-M were tested in a container with a membrane and MB solution. The initial MB solution concentration was $50 \mathrm{mg} \mathrm{L}^{-1}$. Subsequent $\mathrm{MB}$ concentrations were calculated using a standard curve that was measured by UV-vis spectrophotometry. The absorbances of pure water,
$1 \mathrm{mg} \mathrm{L}{ }^{-1}, 3 \mathrm{mg} \mathrm{L}^{-1}, 5 \mathrm{mg} \mathrm{L}^{-1}, 7 \mathrm{mg} \mathrm{L}^{-1}$ and $9 \mathrm{mg} \mathrm{L}^{-1} \mathrm{MB}$ solutions at $665 \mathrm{~nm}$ were measured and a concentrationabsorbance standard curve was plotted.

The photocatalytic performance of all membranes was tested for different irradiations (darkness, UV and sunlight) as shown in Fig. 7. Fig. 7(a) shows that in darkness, the MB absorption capability of all membranes is almost the same, whereas TG-M and NTG-M have a slightly higher absorption capability because of the participation of GO. The GO structure provides a high specific surface area that results in good adsorption
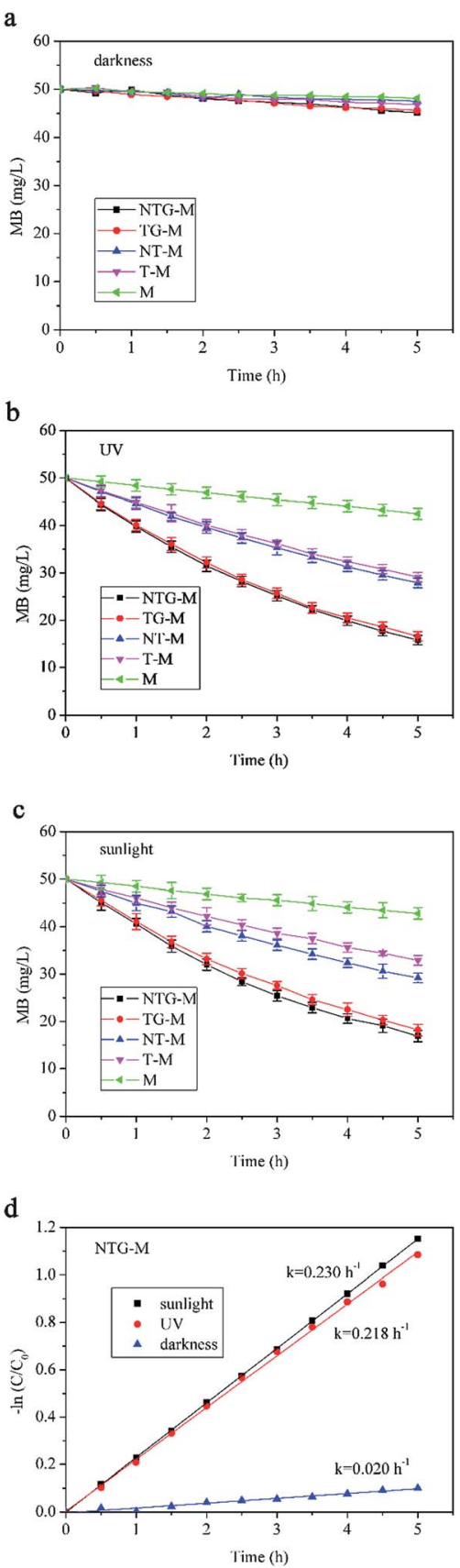

Fig. 7 Residual MB concentration treated by $M, T-M, N T-M, T G-M$ and NTG-M in (a) darkness, (b) UV and (c) sunlight. (d) MB degradation kinetics for NTG-M in darkness, UV and sunlight. $C_{0}$ is the initial MB concentration $\left(50 \mathrm{mg} \mathrm{L}^{-1}\right), C$ is $\mathrm{MB}$ concentration at any time $t$. 
performance. However, the membrane or GO absorption is too low to have a significant effect on the MB removal.

A comparison of NTG-M and NT-M (Fig. 7(b) and (c)) shows that NTG grafting improves photocatalytic performance in UV light and sunlight significantly, just as reported by Gao, ${ }^{25}$ the presences of GO is efficient in improving photocatalytic performance, because GO has an excellent electrical conduction ability and forms an impurity level in $\mathrm{TiO}_{2}$. The $\mathrm{MB}$ concentration in a pure membrane is reduced continuously in UV light and in sunlight. It is expected that MB exhibits a degradation performance in light without catalyst. NTG-M and TG-M have almost the same photocatalytic performance in UV, whereas NTG-M performs better than TG-M in sunlight, which indicates that nitrogen doping did not have a significant impact in UV light but it did in sunlight. Nitrogen doping reduces the $\mathrm{TiO}_{2}$ forbidden band width, and thus $\mathrm{TiO}_{2}$ has a longer response range for light.

The kinetics of MB photodegradation was calculated using a first-order reaction kinetics equation:

$$
-\ln \left(C / C_{0}\right)=k t
$$

$C_{0}\left(\mathrm{mg} \mathrm{L}^{-1}\right)$ is the initial concentration, $C\left(\mathrm{mg} \mathrm{L}^{-1}\right)$ is the $\mathrm{MB}$ concentration at any time $t, k\left(\mathrm{~h}^{-1}\right)$ is the reaction rate constant and $t(\mathrm{~h})$ is the time. ${ }^{38,39}$ Fig. $7(\mathrm{~d})$ summarizes the MB photodegradation kinetics of NTG-M in darkness, UV and sunlight. The kinetics for NTG-M in sunlight is $\sim 4 \%$ faster than in UV, which implies that NTG-M is better utilized in sunlight.

\subsection{Synergistic effect of photocatalysis and filtration}

The synergistic effect of photocatalysis and filtration was tested in the equipment shown in Fig. 1(b). The membrane was placed on the collet with a light source from above to provide energy to power the electron transition. Under nitrogen pressure, MB solution in the feed tank was transported continuously into the photocatalytic membrane reactor. Because of the synergistic effect of photocatalysis and filtration, filtered water flowed out the reactor, whereas $\mathrm{MB}$ was intercepted and photodegraded simultaneously.

We used a photocatalytic filtration concentration $\left(C_{\mathrm{pf}}, \mathrm{mg}\right.$ $\mathrm{L}^{-1}$ ) as a numerical representative for the MB photodegradation ability:

$$
C_{\mathrm{pf}}=\frac{\left[C_{0}\left(V_{\mathrm{r}}+V_{t}\right)-C_{t 1} V_{\mathrm{r}}-C_{t 2} V_{t}\right]}{V_{t}}
$$

$C_{0}\left(\mathrm{mg} \mathrm{L}^{-1}\right)$ is the initial MB concentration, $C_{t 1}\left(\mathrm{mg} \mathrm{L}^{-1}\right)$ is the instantaneous $\mathrm{MB}$ concentration in the reactor at any time $t(\mathrm{~h})$, $C_{t 2}\left(\mathrm{mg} \mathrm{L}^{-1}\right)$ is the instantaneous $\mathrm{MB}$ concentration of filtered water at any time $t, V_{\mathrm{r}}(\mathrm{L})$ is the reactor volume and $V_{t}(\mathrm{~L})$ is the filtered water volume. We approximate the initial MB concentration in the feed tank as constant despite the increase or decrease in $\mathrm{MB}$ concentration in the reactor. In this study, the initial concentration of MB solution in the feed tank was $50 \mathrm{mg}$ $\mathrm{L}^{-1}$, the system pressure was maintained at $0.04 \mathrm{MPa}$ during the tests and the reactor volume was $0.05 \mathrm{~L}$.

Fig. 8 shows the synergistic effect of photocatalysis and filtration of NTG-M and $M$ in sunlight. Fig. 8(a) shows that all of a

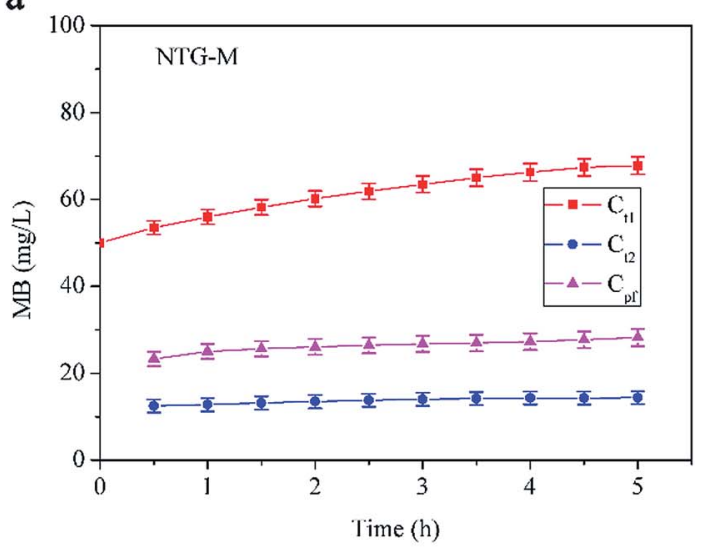

b
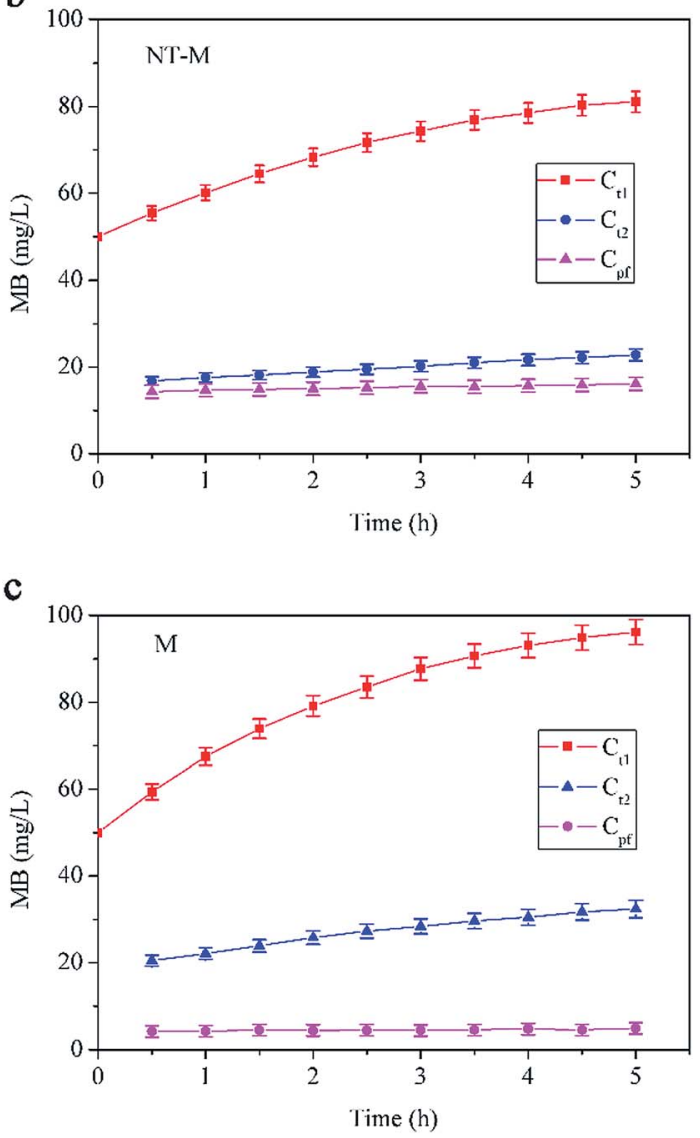

Fig. $8 \mathrm{MB}$ concentration in the reactor, filtered water MB concentration and photocatalytic filtration concentration for (a) NTG-M, (b) $N T-M$ and (c) $M$ in sunlight. The initial MB concentration in the reactor was $50 \mathrm{mg} \mathrm{L}^{-1}$.

the NTG-M concentration curves exhibit an increasing trend, whereas the concentration curve in the reactor exhibits the largest increase. At $0.5 \mathrm{~h}$, the photocatalytic filtration concentration is $\sim 23.3 \mathrm{mg} \mathrm{L}^{-1}$, which means that $1 \mathrm{~L}$ water is filtered and $22.3 \mathrm{mg} \mathrm{MB}$ will be photodegraded. Because the total $\mathrm{MB}$ concentration $\left(35.8 \mathrm{mg} \mathrm{L} \mathrm{L}^{-1}\right.$ ) of the photocatalytic filtration concentration and filtered water $\left(12.5 \mathrm{mg} \mathrm{L}^{-1}\right)$ is lower than the initial $\mathrm{MB}$ concentration $\left(50 \mathrm{mg} \mathrm{L}^{-1}\right)$, the $\mathrm{MB}$ concentration in 
the reactor will increase. Because the MB concentration in the reactor increases, the photocatalytic rate will increase according to first-order reaction kinetics, and thus, the photocatalytic filtration concentration will increase. Fig. 8(b) shows the concentration curves of $\mathrm{M}$; the trend in basic concentration curves in the reactor and filtered water are the same as NTG-M. Because the photocatalytic performance of NT-M is lower than NTG-M, the photocatalytic filtration concentration is lower than NTG-M, thus the MB concentration in the reactor increase faster than NTG-M. Fig. 8(c) shows the concentration curves of $\mathbf{M}$; the trend in basic concentration curves in the reactor and filtered water are also the same as NTG-M, but its degree of change is larger. Without a photocatalyst, the photodegradation of $\mathrm{MB}$ can almost be ignored as the photocatalytic filtration concentration of $\mathrm{M}$ is less than $5 \mathrm{mg} \mathrm{L}^{-1}$. NTG-M exhibits a higher removal of $\mathrm{MB}$ because its $\mathrm{MB}$ concentration of filtered water is lower than $M$ because of its photocatalytic performance. Because the MB concentration of NTG-M in the reactor is lower than $\mathbf{M}$, the possibility of membrane pollution is reduced significantly. Thus, under the synergistic effect of photocatalysis and filtration, the entire MB removal ability of NTG-M exhibits a great improvement.

\subsection{Recycle performance}

To evaluate the recycle performance of NTG-M, we measured the pure water flux of NTG-M continuously and tested the photocatalytic performance of NTG-M repeatedly. The pure water flux of NTG-M was tested in the system as illustrated in Fig. 1(a) at $0.1 \mathrm{MPa}$ and at room temperature. Similarly, the photocatalytic performance of NTG-M were tested in a container with a membrane and MB solution in sunlight, the initial $\mathrm{MB}$ solution concentration was $50 \mathrm{mg} \mathrm{L}^{-1}$ and adjusted to $50 \mathrm{mg} \mathrm{L}^{-1}$ every $3 \mathrm{~h}$.

As shown in Fig. 9, the residual MB concentration is $\sim 25 \mathrm{mg}$ $\mathrm{L}^{-1}$ after being photodegraded by NTG-M for the first $3 \mathrm{~h}$ in sunlight, and NTG-M shows the similar photocatalytic performance in the second $3 \mathrm{~h}$ while the residual $\mathrm{MB}$ concentration is raised gradually in the following tests. At the end of the eighth

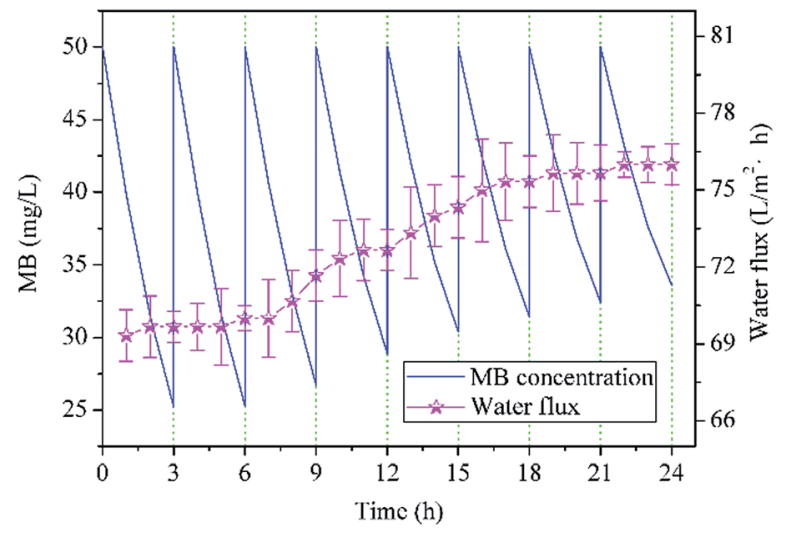

Fig. 9 Residual MB concentration treated by NTG-M in sunlight (the initial $M B$ concentration was always $50 \mathrm{mg} \mathrm{L}^{-1}$ ) and pure water flux of NTG-M at $0.1 \mathrm{MPa}$ at room temperature.
$3 \mathrm{~h}$, the residual $\mathrm{MB}$ concentration is $\sim 33.5 \mathrm{mg} \mathrm{L}^{-1}$, its photodegradation ability decreases $\sim 34 \%$ compare with which in the first $3 \mathrm{~h}$. This is possibly caused by the loss of photocatalyst or MB deposition in the photocatalyst. The pure water flux of NTG-M is also shown in Fig. 9, its changing curve exhibits a weak increase as the increasement of pure water flux is $~ 9 \%$ after $24 \mathrm{~h}$. Because the photocatalyst on the membrane surface has an obstructive performance, we guess that a small amount of photocatalyst has lost and thus pure water flux raise. Considering above two kinds of test results, we think that a small part of the photocatalyst on NTG-M is losing during use, but the recyclability of photocatalyst has a great improvement compare with powder photocatalyst.

\section{Conclusions}

An ultrafiltration membrane grafted by $\mathrm{N}-\mathrm{TiO}_{2} /$ graphene oxide (NTG-M) was prepared. SEM images show membrane surfaces that are covered with flakes. FT-IR signals of oxygen-containing functional groups show a graphene oxide success load on the membrane surface. XPS signals of the peaks and elemental composition of the nitrogen and titanium reveal successful $\mathrm{N}$ doping on $\mathrm{TiO}_{2}$ and NT loading on the membrane surface. The surface roughness as tested by AFM shows that NTG-M exhibits a rougher surface, which is advantageous to its photodegradation. Contact-angle measurements show that NTG-M has a lower water-contact angle, which implies better hydrophilicity. However, a lower pure water flux of NTG-M was tested by using the ultrafiltration cup system because of obstructive performance of the NTG, indicating membrane grafted by N$\mathrm{TiO}_{2}$ /graphene oxide is not all advantages. By testing its photocatalytic performance, we believe that $\mathrm{N}$-doping and graphene oxide have a positive effect on the $\mathrm{TiO}_{2}$ photocatalytic performance, and NTG-M is used better in sunlight than in UV light. By testing the synergistic effect of photocatalysis and filtration, we conclude that NTG-M has a better MB removal ability and antifouling ability because of its photocatalytic performance. Furthermore, the recyclability of the NTG-M has a great improvement compare with powder photocatalyst.

\section{Acknowledgements}

This work was supported by the National Natural Science Foundation of China (No. 51678213 and No. 51578209), and A Project Funded by the Priority Academic Program Development of Jiangsu Higher Education Institutions.

\section{References}

1 G. Moon, D. Kim, H. Kim, A. D. Bokare and W. Choi, Environ. Sci. Technol. Lett., 2014, 1, 185-190.

2 M. N. Chong, B. Jin, C. W. K. Chow and C. Saint, Water Res., 2010, 44, 2997-3027.

3 W. Qian, P. A. Greaney, S. Fowler, S. Chiu, A. M. Goforth and J. Jiao, ACS Sustainable Chem. Eng., 2014, 2, 1802-1810.

4 D. Kanakaraju, J. Kockler, C. A. Motti, B. D. Glass and M. Oelgemöller, Appl. Catal., B, 2015, 166-167, 45-55. 
5 F. Pei, Y. Liu, S. Xu, J. Lü, C. Wang and S. Cao, Int. J. Hydrogen Energy, 2013, 38, 2670-2677.

6 M. Pelaez, N. T. Nolan, S. C. Pillai, M. K. Seery, P. Falaras, A. G. Kontos, P. S. M. Dunlop, J. W. J. Hamilton, J. A. Byrne, K. O'Shea, M. H. Entezari and D. D. Dionysiou, Appl. Catal., B, 2012, 125, 331-349.

7 R. Wang, Q. Wu, Y. Lu, H. Liu, Y. Xia, J. Liu, D. Yang, Z. Huo and X. Yao, ACS Appl. Mater. Interfaces, 2014, 6, 2118-2124.

8 X. Chen and S. S. Mao, Chem. Rev., 2007, 107, 2891-2959.

9 W. Sangkhun, L. Laokiat, V. Tanboonchuy, P. Khamdahsag and N. Grisdanurak, Superlattices Microstruct., 2012, 52, 632-642.

10 H. Zhang, G. Wang, D. Chen, X. Lv and J. Li, Chem. Mater., 2008, 20, 6543-6549.

11 H. Zhang, L. Guo, D. Wang, L. Zhao and B. Wan, ACS Appl. Mater. Interfaces, 2015, 7, 1816-1823.

12 H. Li, Y. Hao, H. Lu, L. Liang, Y. Wang, J. Qiu, X. Shi, Y. Wang and J. Yao, Appl. Surf. Sci., 2015, 344, 112-118.

13 G. K. Mor, K. Shankar, M. Paulose, O. K. Varghese and C. A. Grimes, Nano Lett., 2006, 6, 215-218.

14 W. Sun, Y. Yu, H. Pan, X. Gao, Q. Chen and L. Peng, J. Am. Chem. Soc., 2008, 130, 1124-1125.

15 C. Gu and C. Shannon, J. Mol. Catal. A: Chem., 2007, 262, 185-189.

16 Y. Liu, S. Wang, S. Xu and S. Cao, Mater. Res. Bull., 2015, 65, 27-35.

17 T. Nguyen-Phan, V. H. Pham, E. W. Shin, H. Pham, S. Kim, J. S. Chung, E. J. Kim and S. H. Hur, Chem. Eng. J., 2011, 170, 226-232.

18 W. Wang, Y. Xie, C. Xia, H. Du and F. Tian, Microchim. Acta, 2014, 181, 1325-1331.

19 D. Zhao, G. Sheng, C. Chen and X. Wang, Appl. Catal., B, 2012, 111-112, 303-308.

20 X. Pan, M. Yang, Z. Tang and Y. Xu, J. Phys. Chem. C, 2014, 118, 27325-27335.

21 S. Liu, H. Sun, S. Liu and S. Wang, Chem. Eng. J., 2013, 214, 298-303.
22 W. Yang, C. Li, L. Wang, S. Sun and X. Yan, Appl. Surf. Sci., 2015, 353, 307-316.

23 G. Xiao, X. Zhang, W. Zhang, S. Zhang, H. Su and T. Tan, Appl. Catal., B, 2015, 170-171, 255-262.

24 L. M. Pastrana-Martínez, S. Morales-Torres, J. L. Figueiredo, J. L. Faria and A. M. T. Silva, Water Res., 2015, 77, 179-190.

25 Y. Gao, M. Hu and B. Mi, J. Membr. Sci., 2014, 455, 349-356.

26 A. Alem, H. Sarpoolaky and M. Keshmiri, J. Eur. Ceram. Soc., 2009, 29, 629-635.

27 Y. Wei, H. Chu, B. Dong, X. Li, S. Xia and Z. Qiang, Desalination, 2011, 272, 90-97.

28 J. Xu, C. Chang, J. Hou and C. Gao, Chem. Eng. J., 2013, 223, 722-728.

29 X. Li, X. Fang, R. Pang, J. Li, X. Sun, J. Shen, W. Han and L. Wang, J. Membr. Sci., 2014, 467, 226-235.

30 X. Huang, K. L. Marsh, B. T. McVerry, E. M. V. Hoek and R. B. Kaner, ACS Appl. Mater. Interfaces, 2016, 8, 1433414338.

31 H. M. Hegab and L. Zou, J. Membr. Sci., 2015, 484, 95-106.

32 M. Kumar, Z. Gholamvand, A. Morrissey, K. Nolan, M. Ulbricht and J. Lawler, J. Membr. Sci., 2016, 506, 38-49.

33 F. Shi, Y. Ma, J. Ma, P. Wang and W. Sun, J. Membr. Sci., 2012, 389, 522-531.

34 M. Safarpour, A. Khataee and V. Vatanpour, J. Membr. Sci., 2015, 489, 43-54.

35 O. C. Compton, B. Jain, D. A. Dikin, A. Abouimrane, K. Amine and S. T. Nguyen, ACS Nano, 2011, 5, 4380-4391.

36 N. A. Almeida, P. M. Martins, S. Teixeira, J. A. Lopes Da Silva, V. Sencadas, K. Kühn, G. Cuniberti, S. Lanceros-Mendez and P. A. A. P. Marques, J. Mater. Sci., 2016, 51, 6974-6986.

37 M. Safarpour, A. Khataee and V. Vatanpour, Ind. Eng. Chem. Res., 2014, 22211943.

38 M. Nie, Y. Yang, Z. Zhang, C. Yan, X. Wang, H. Li and W. Dong, Chem. Eng. J., 2014, 246, 373-382.

39 A. E. Cassano and O. M. Alfano, Catal. Today, 2000, 58, 167197. 